\title{
Mapping Narrative Content and Structure in Online Atlases
}

\author{
Gareth Baldrica-Franklin ${ }^{\mathrm{a}}$ \\ ${ }^{a}$ University of Wisconsin-Madison, baldricafran@wisc.edu
}

Keywords: Online Atlases, Content Analysis, Narrative, Storytelling

Problem: Atlases are united by narrative potential (Buckley 2003). While some have theorized that atlases convey narrative in the syntax between their maps (Pearce 2008), overall, there has been little study of how atlas narratives could function. This is particularly true for digital and online atlases, which have different possibilities of sequencing and structure than print. Storytelling, in any capacity, has a dual nature within atlases: individual maps can tell stories, and these maps can also coalesce into a larger story (Pearce 2008). This research will examine current trends in online atlas narratives on both levels, analyzing structure and individual map content within 20 digital online atlases.

Background: An atlas is a communication device - a selection of maps related by theme, or region, and organized into a volume (Buckley 2003). While print atlases have existed, in one form or another, for hundreds of years, the advent of digital atlases in the 1980s prompted reconsideration of an atlas' core qualities (Ramos and Cartwright 2006). Some digital atlases were view-only, scanned versions of their print counterparts, others were atlas information systems (AIS), which contained multiple data views and advanced analytical capabilities, while many more existed somewhere between these two poles (Ramos and Cartwright 2006). Ultimately, there was agreement that all atlases, from print to digital, were united in their ability to tell stories (Buckely 2003, Ramos and Cartwright 2006).

Broadly, storytelling is a method of communicating human experience through abstracted accounts of people, events, and places (Roth 2021). In recent years, the relationship between storytelling and cartography has received increased attention and study (Pearce 2008, Caquard 2013, Denil 2017). Seen as an avenue for hybridization in the discipline, cartographic storytelling combines theory and practice from arts, sciences, and the humanities. Key to storytelling is narrative, or narrative structure, which refers to how a story is organized. In other words, how elements of a story are arranged and presented to the reader. Structure can be linear, and follow a temporal, or highly ordered series of events (Roth 2021), or it can be nonlinear, and provide little or no explicit ordering (Keturakis 2015). In visual storytelling, structure often follows a linear pattern, conforming to narrative conventions such as the three-act structure.

While print atlases could be logically organized as linear narratives (though not necessarily), online atlases, a subset of digital atlases hosted on the web, have structural characteristics unique to the online medium. As series of interconnected webpages, online atlases have inherently nonlinear qualities, as each individual user could have a unique narrative experience every time they interact. Thus, online atlases could be reconsidered as Interactive Digital Narratives (IDNs) - narratives that change according to user input (Koenitz 2015). IDNs differ from other narrative forms due to the inclusion of choice presented to the user. In an online atlas, choice could manifest as map selection, as well as how users can navigate between different maps in the atlas, and how these choices contribute to the broader narrative. IDN authoring platforms often provide a visual schema to map choices and consequences. This is known as the graph view, and is used to visualize the relationship between individual story nodes (or scenes) to one another. When a story is complete, analysis of the graph view can provide insight about the structure of the story (Koenitz 2012).

Methods: The goal of this content analysis was to uncover current practices in narrative and structural design of online atlases. This included both narrative elements used within individual pages, as well as overall atlas structure. I obtained a sample of online atlases through search engines. To be included in the analysis, atlases had to have a map emphasisfinished maps were the primary means of communication within the site, as opposed to data portals, or GIS interfaces. Atlases had to contain multiple maps, and had to have a theme or region, to exclude general online collections of maps or data (Buckley 2003, Ramos and Cartwright 2006). At least one map within the atlas had to be interactive, and not a view-only scan or PDF of a print atlas. Lastly, atlases had to have a non-expert audience, including edited data not shown in its raw, unprocessed form (Ramos and Cartwright 2006). Overall, 114 online atlases were considered, with Table 1 listing the final sample of 20 meeting inclusionary criteria.

Once I made the selection, atlases were first mapped according to their webpage structure (Figure 1). I then compared the resulting graph maps with basic statistics, such as the total number of pages and maps. Atlases were also coded based on their structure, borrowing codes from Koenitz (2012) that consider the shape of an IDN, and adapting them for online atlas application. These codes included the clustering of pages, the amount of entry points, and how individual maps were connected to one another. After atlas structure was mapped, individual pages within the atlas were coded according to principles of cartographic storytelling, including aspects of narrative elements, structure, navigation, and data presentation (Stolper et al. 2016, Roth 2021). 
Preliminary Results: An example graph map of an online atlas used for analysis can be seen in Figure 1. In these diagrams, each webpage in the atlas was represented as a node, with links to other pages shown as lines between nodes. The example shown in Figure 1 was multi-clustered, meaning there were multiple distinct groups of pages, a common practice. Overall, a wide variety of structures were represented across the sample, from intricate branching webs with many interconnected pages, to those with strict limits on navigation and progression through content. Additionally, while full analysis of individual atlas maps is ongoing, early results suggests a roughly even split in the presence/absence of explicit story maps in each atlas across the sample.

\begin{tabular}{lll} 
Atlas Title & & \\
\hline Atlas of Economic Complexity & Atlas of the Salish Sea & Wyoming Student Atlas \\
Atlas of Canada & Early Islamic Empire at Work & Atlas of the Pacific Northwest \\
Environmental Justice Atlas & IMPACT2C Web Atlas & Oregon Water Atlas \\
Atlas of Sustainable Development Goals & Geospatial Conservation Atlas & Global Refugee Atlas \\
Atlas of the Historical Geography of the U.S & Marine Protection Atlas & American Panorama \\
Mapping Past Societies & Atlas of Surveillance & Digital Atlas of Innovations \\
North Dakota Digital Atlas & Forest Atlas of the U.S & \\
\hline
\end{tabular}

Table 1. Sample of Online Atlases
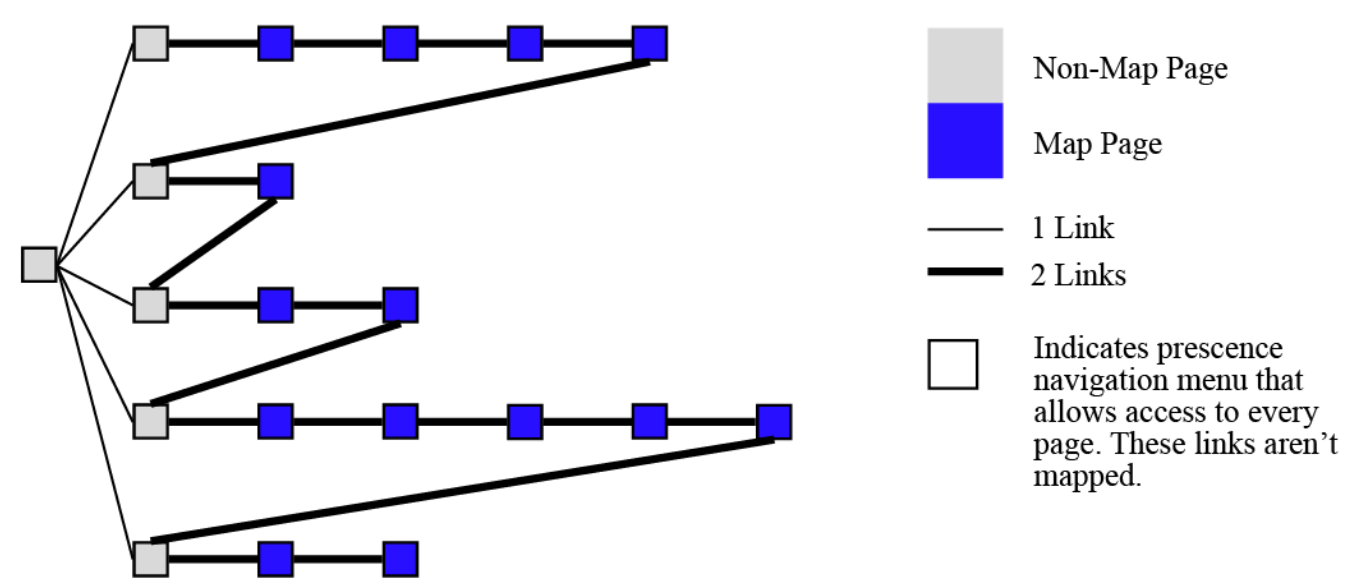

Figure 1. Graph map of the Forest Atlas of the U.S.

\section{References}

Buckley, A. (2003). Atlas Mapping in the 21st Century. Cartography and Geographic Information Science, 30(2), 149158.

Caquard, S. (2013). Cartography I: Mapping narrative cartography. Progress in Human Geography, 37(1), $135-144$.

Denil, M. (2017) "Storied Maps" Cartographic Perspectives (84) pp.5-22

Keturakis, S. (2015). Topography of a Dream, or Some Features of Nonlinear Narrative. Creativity Studies, 9(1), 5363.

Koenitz, H., \& Chen, K.-J. (2012). Genres, Structures and Strategies in Interactive Digital Narratives - Analyzing a Body of Works Created in ASAPS. In D. Oyarzun, F. Peinado, R. M. Young, A. Elizalde, \& G. Méndez (Eds.), Interactive Storytelling (Vol. 7648, pp. 84-95). Springer Berlin Heidelberg.

Koenitz, H. (2015). Design Approaches for Interactive Digital Narrative. In H. Schoenau-Fog, L. E. Bruni, S. Louchart, \& S. Baceviciute (Eds.), Interactive Storytelling (Vol. 9445, pp. 50-57). Springer International Publishing.

Pearce, M. W. (2008). Framing the Days: Place and Narrative in Cartography. Cartography and Geographic Information Science, 35(1), 17-32.

Ramos, C. da S., \& Cartwright, W. (2006). Atlases from Paper to Digital Medium. In E. Stefanakis, M. P. Peterson, C. Armenakis, \& V. Delis (Eds.), Geographic Hypermedia (pp. 97-119). Springer Berlin Heidelberg

Roth, R. E. (2021). Cartographic Design as Visual Storytelling: Synthesis and Review of Map-Based Narratives, Genres, and Tropes. The Cartographic Journal, 1-32.

Stolper, C. D., Lee, B., Riche, N. H., \& Stasko, J. (n.d.). Emerging and Recurring Data-Driven Storytelling Techniques: Analysis of a Curated Collection of Recent Stories. 14. 